Physica C 152 (1988) 247-250

North-Holland, Amsterdam

\title{
AC LOSSES AND CRITICAL CURRENT DENSITY OF SUPERCONDUCTING $\mathrm{GdBa}_{2} \mathrm{Cu}_{3} \mathrm{O}_{7-x}$
}

\author{
M. CISZEK, J. OLEJNICZAK, E. TROJNAR, A.J. ZALESKI and J. KLAMUT \\ Institute for Low Temperature and Structure Research, Polish Academy of Sciences, 53-529 Wroclaw, Prochnika Str. 95, Poland
}

A.J.M. ROOVERS and L.J.M. VAN DE KLUNDERT

University of Twente, Department of Applied Physics, P.O. Box 217, 7500 AE Enschede, The Netherlands

Received 17 March 1988

Revised manuscript received 8 April 1988

\begin{abstract}
Energy losses occurring in a cylindrical sample of $\mathrm{Gd}-\mathrm{Ba}-\mathrm{Cu}-\mathrm{O}$, subjected to an external $\mathrm{AC}$ magnetic field were examined. The loss dependence on the amplitude of the magnetic induction exhibits two stages of flux penetration into the superconductor. Critical current densitities for both stages of penetration were examined and an explanation for such behaviour is proposed. Support for this point of view is obtained by measurements on pulverized sample material. All measurements were performed at a temperature of $4.2 \mathrm{~K}$ and in absence of a background field.

Analysis of the data provides two critical current densities: an inter-granular critical current density at weak alternating magnetic fields and an intra-granular critical current density at higher magnetic fields. The intra-granular critical current density is at least two orders of magnitude larger than the inter-granular one.
\end{abstract}

\section{Introduction}

The practical large-scale applications of high- $T_{\mathrm{c}}$ superconductors are strongly limited by the magnitude of the electrical current which can be lossless transported through the material. The existing results concerning the critical current densities in $\mathrm{YBa}_{2} \mathrm{Cu}_{3} \mathrm{O}_{7-x}$ samples are not too encouraging. The critical current densities, obtained from critical current measurements, are of the order of $10^{7} \mathrm{Am}^{-2}$, which is at least two orders of magnitude too low for practical applications. However, the critical current derived from magnetization measurements proved the potential applicability of the new superconductors [1-5]. The reason of this discrepancy between the transport and magnetization $J_{\mathrm{c}}$ values are most probably weak links between the superconducting regions in granular superconductors [6-9]. The aim of our experiments was too examine this phenomenon and to estimate the values of the intrinsic and extrinsic critical current densities.

\section{Sample preparation}

Superconducting specimens of $\mathrm{Gd}-\mathrm{Ba}-\mathrm{Cu}-\mathrm{O}$ were prepared through the usual solid state reaction of fine powders of pure $\mathrm{Gd}_{2} \mathrm{O}_{3}, \mathrm{BaCO}_{3}$ and $\mathrm{CuO}$. The mixed powders were pressed into pellets and calcined for 8 hours at $940^{\circ} \mathrm{C}$ in air and then three times at $900^{\circ} \mathrm{C}$ for 5 hours with intermediate grinding steps. Finally, after pulverization, the powder was pressed into a cylindrical rod of $5 \mathrm{~mm}$ in diameter and $11 \mathrm{~mm}$ in length, at a pressure of approximately $11 \mathrm{kbar}$, and heated at $900^{\circ} \mathrm{C}$ for several hours in a flowing atmosphere.

The X-ray powder diffraction patterns indicated a single phase orthorhombic-distorted structure, the same as that of $\mathrm{YBa}_{2} \mathrm{Cu}_{3} \mathrm{O}_{7-x}$ samples. The resistive transition, measured with a standard four-lead technique, had a midpoint of transition at $92.6 \mathrm{~K}$ and its width was $2.4 \mathrm{~K}$. 


\section{AC loss measurements}

\subsection{Experimental technique}

The sample, with a pick-up coil consisting of 100 turns of $20 \mu \mathrm{m}$ copper wire wound directly on the central part of the cylinder, was placed axially inside a superconducting solenoid producing a sinusoidally varying magnetic field.

For small amplitudes of the external magnetic induction the losses were measured by means of a lockin amplifier technique. The hysteresis loops were taken from the oscilloscope, where on the $x$ and $y$ axis were plotted respectively the signals proportional to the amplitude $b_{0}$ and the integrated voltage from the pick-up coil.

For larger amplitudes of the applied field the losses in the sample were determined using a computercontrolled measuring system as previously described in ref. [10]. The set-up was adjusted for the cylindrical shaped sample by rcplacing the installed pickup coil by one attached directly to the surface of the sample.

\subsection{Results}

The dependence of the AC losses $W$ on the amplitude of the alternating external magnetic induction $b_{0}$ for the cylindrical shaped sample is shown in fig. 1. The plot can be divided into three regions.

In the first one, the magnetic flux starts to penetrate into the sample, already for amplitudes $b_{0}$ of the order of $1 \mathrm{mT}$. The losses increase with approximately the fourth power of the amplitude $b_{0}$.

At a certain value of $b_{0}$ the losses start to saturate and remain nearly constant for some interval of the amplitude of the magnetic induction. For this region a frequency dependence of the losses can be noticed. It means probably that eddy current losses, apart from the hysteretic ones, play some role in the material investigated.

In the third region of the plot in fig. 1, for the highest available values of $b_{0}$ in our experiment, the losses are not dependent of the frequency, i.e. they are purely hysteretic in nature. In this region the losses exhibit a field dependence approximately as $W \sim b_{0}^{3}$.

The hysteresis loops $(\Phi-b)$ for different values of $b_{0}$ equal to $3,7,12$, and $100 \mathrm{mT}$, observed on the

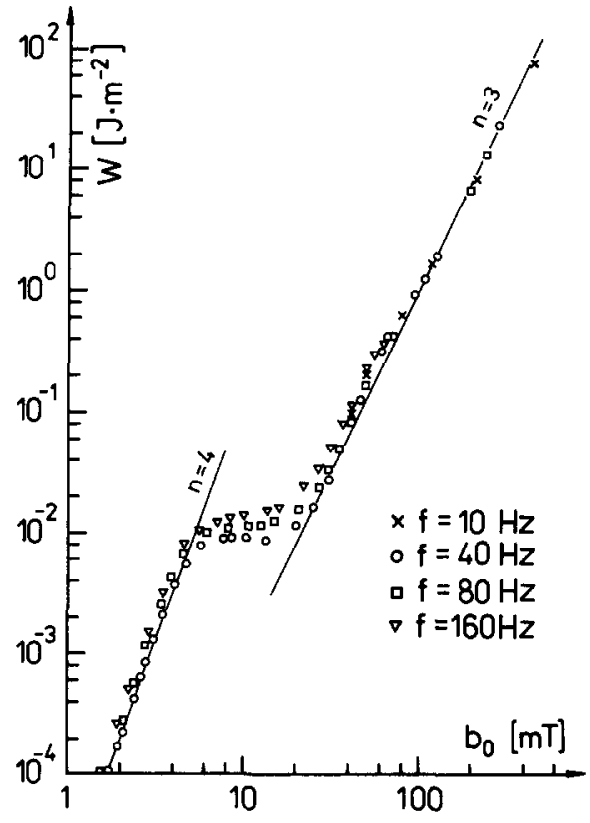

Fig. 1. The dependence of $\mathrm{AC}$ losses of the cylindrical shaped sample on the amplitude of the external magnetic induction for different frequencies. The solid line with the slope $n=4$ corresponds to calculations according to ref. [15] for $\alpha=1.1 \times 10^{6}$ $\mathrm{TAm}^{-2}$ and $B_{0}=0.5 \mathrm{mT}$.

oscilloscope are presented in fig. 2. For low amplitudes of the magnetic induction, the hysteresis loops are typical for type-II superconductors. With increasing $b_{0}$ the loop increases as well. However, from a certain value of $b_{0}$ its enclosed area remains constant. Further increase of the the amplitude $b_{0}$ does not affect the area of the loops (the "plateau" region in fig. 1); only straight line "tails" occur with steady increasing length. From an amplitude of about 20 $\mathrm{mT}$ a further increase of the hysteresis loop area occurs. This corresponds to the region where the losses are proportional to the third power of the magnetic field amplitude $b_{0}$.

Figure 3 shows the result of the loss measurements on pulverized $\mathrm{GdBa}_{2} \mathrm{Cu}_{3} \mathrm{O}_{7-x}$. The results coincide with those of the cylindrical sample for large values of the amplitude of the magnetic field $b_{0}$ (the third region in the plot of fig. 1). The "plateau" region however has disappeared. 


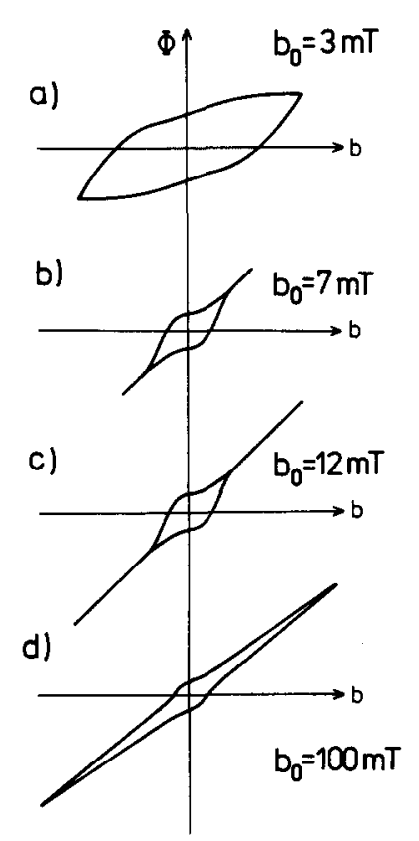

Fig. 2. A set of magnetic hysteresis loops taken from an oscilloscope for different values of the amplitude of the external magnetic induction. The drawings are not in scale, $\Phi$ values are in arbitrary units. The measurements are performed on the cylindrical shaped sample.

\section{Discussion}

From our point of view the existence of three different regions in the $W\left(b_{0}\right)$ dependence can be explained by assuming that the sample consists of grains of material with high critical parameters interconnected by weak links. When increasing the $\mathrm{AC}$ magnetic field amplitude $b_{0}$, magnetic flux starts to penetrate the weak links first. For small magnetic field amplitudes the losses are proportional to the fourth power of the amplitude of the magnetic field. This can be explained by a strong field dependence of the critical current density in the vicinity of zero field. For amplitudes sufficiently high, the magnetic field penetrates the weak links and drives them into the normal state. It is seen as a "plateau" in fig. 1, and as hysteresis loops with the same surface areas but with longer "tails". In this region the AC losses are frequency dependent, which is characteristic for normal metals. For higher amplitudes $b_{0}$ the $\mathrm{AC}$ losses are connected with properties of the superconducting material inside the grains. For this region

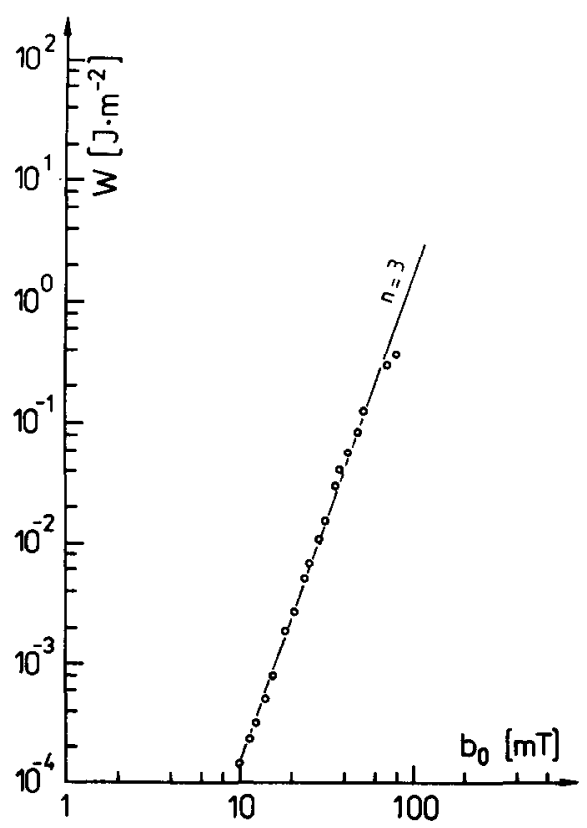

Fig. 3. AC losses of pulverized $\mathrm{GdBa}_{2} \mathrm{Cu}_{3} \mathrm{O}_{7-x}$ as a function of the amplitude of an alternating magnetic field.

the magnetic field dependence of $J_{c}$ is rather weak and its value is connected with flux pinning. Also for this region the anisotropic properties of the material within a grain may have a strong influence on the critical current of the polycrystalline samples [6].

In the first region of the plot of fig. 1 , the $W\left(b_{0}\right)$ dependence can be described by using the Kim model, in which the $J_{\mathrm{c}}(B)$ dependence is written as $J_{\mathrm{c}}(|B|)=\alpha\left(B_{0}+|B|\right)$. The value of the critical current density can be estimated from the AC loss measurements by fitting the parameters $\alpha$ and $B_{0}$ using the expressions derived by Fournet and Mailfert [11]. In ref. [11] loss calculations are presented using a slab model for different field dependences of the critical current density. The best fit with the experimental data was obtained for the values $\alpha=1.1 \times 10^{6} \mathrm{ATm}^{-2}$ and $B_{0}=0.5 \mathrm{mT}$. The value of the constant $B_{0}$ supports our finding that the $H_{\mathrm{c} 1}$ value is very low (it should be of the order of $B_{0}$ [12]) and that $J_{\mathrm{c}}$ is very strongly field dependent. The critical current density $J_{\mathrm{c}}$ derived for these constants equals about $2 \times 10^{7} \mathrm{Am}^{-2}$ for $B=0$. The low value of the constant $B_{0}$, equal to about $1 \mathrm{mT}$ for a 
$\mathrm{Y}-\mathrm{Ba}-\mathrm{Cu}-\mathrm{O}$ compound, was also reported in ref. [13].

For larger amplitudes of the applied magnetic field the loss behaviour of the cylindrical shaped sample corresponds to the one of the pulverized material. Therefore it is plausible to assume that the magnetization is dominated by shielding currents inside the grains. If in the above-mentioned theory isolated "grains" are considered the intra-granular critical current density can be estimated. Calculation of the critical current density from the loss measurements requires that the size of the grains is known. From our measurements the magnitude of the critical current density is estimated to be $10^{10}-10^{11} \mathrm{Am}^{-2}$, assuming a grain size in the order of micrometers [14]. For instance, assuming a grain size of $10 \mu \mathrm{m}$ yields a good fit for $\alpha=5 \times 10^{10} \mathrm{ATm}^{-2}$ and $B_{0}=0.5 \mathrm{~T}$ in the third region of the plot in fig. 1.

\section{Conclusions}

The results of our experiments show a difference between the intrinsic and extrinsic critical current densities of at least two orders of magnitude. From our explanation it is seen that to obtain larger extrinsic critical current densities it is necessary to improve the preparation technique in order to avoid weak links between the grains and improve the contacts between them. Therefore a further study to the origin of those weak links is necessary.
References

[1] Y. Yamada, N. Fukushima, S. Nakajama, H. Yoshino and S. Murase, Jpn. J. Appl. Phys. 26 (1987) L865.

[2] H. Kumakura, K. Togano, M. Fukutami, M. Uehara and K. Tachikawa, Jpn. J. Appl. Phys. 26 (1987) L655.

[3] H. Kumakura, M. Uehara, Y. Yoshida and K. Togano, Phys. Lett. A 124 (1987) 367.

[4] Gang Xiao, F.H. Streitz, A. Gavrin, M.Z. Cieplak, J. Childress, Ming Lu, A. Zwicker and C.L. Chien, Phys. Rev. B 36 (1987) 2382.

[5] K. Rogacki, C. Sulkowski, A.J. Zaleski, Z. Bukowski, M. Ciszek, R. Horyn, E. Trojnar and J. Klamut, Phys. Status Solidi a 102 (1987) K117.

[6] H. Kupfer, I. Apfelstedt, W. Schauer, R. Flukiger, R. MeierHirmer and H. Wuhl, Z. Phys. B 69(2/3) (1987) 159.

[7] D. Dew-Hughes, Philos. Mag. B 55(4) (1986) 459.

[8] R.B. Goldfarb, A.F. Clark, A.I. Braginski and A.J. Panson, Cryogenics 27 (1987) 475.

[9] S. Senousi, M. Oussena, M. Ribault and G. Collin, Phys. Rev. 36 (1987) 4003.

[10] A.J.M. Roovers, P.P.E. Fornerod, W. Heida and L.J.M. van de Klundert, IEEE Trans. Magn. MAG-23(2) (1987) 1736.

[11] G. Fournet and A. Mailfert, J. Phys. (France) 31 (1970) 357.

[12] Y.B. Kim, C.F. Hempstead and A.R. Strnad, Phys. Rev. Lett. 9 (1962) 306.

[13] L.J.M. van de Klundert, P. Out, B. ten Haken, G.J.M. Velders, D.H.A. Blank and W.S.J. Uijttewaal, in: Proc. European Workshop on High- $T_{c}$ Superconducitvity and Potential Applications, Genova, Italy (1987), p. 409 (paper P-69).

[14] M.E. McHenry, J. McKittrick, S. Sasayama, V. Kwapong, R.C. O'Handley and G. Kalonji, Phys. Rev. B 37 (1988) 623.

[15] D.K. Finnemore, J.E. Ostenson, L. Ji, R.W. McCallum and J.R. Clem, preprint. 